\title{
RESEARCH
}

Open Access

\section{Cerebrovascular disease influences functional and structural network connectivity in patients with amnestic mild cognitive impairment and Alzheimer's disease}

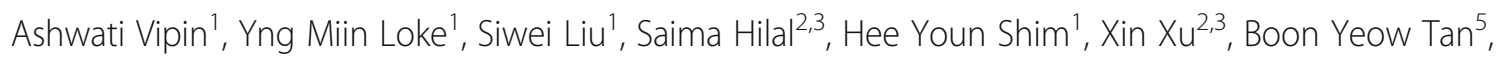
Narayanaswamy Venketasubramanian ${ }^{6}$, Christopher Li-Hsian Chen ${ }^{2,3}$ and Juan Zhou ${ }^{1,4^{*}}$ (D)

\begin{abstract}
Background: Patients with amnestic mild cognitive impairment (aMCl) and Alzheimer's disease (AD) show functional and structural connectivity alterations in the default mode network (DMN) while cerebrovascular disease (CeVD) shows functional and structural connectivity changes in the executive control network (ECN). Such disruptions are associated with memory and executive function impairment, respectively. Concurrent AD and CeVD pathology is associated with a higher rate of cognitive decline and differential neurodegenerative patterns. Together, such findings are likely reflective of different underlying pathology in AD with and without CeVD. However, few studies have examined the effect of CeVD on network functional connectivity (task-free functional magnetic resonance imaging (fMRI)) and structural connectivity (diffusion MRI) of the DMN and ECN in aMCl and AD using a hypothesis-driven multiple seed-based approach.
\end{abstract}

Methods: We examined functional and structural connectivity network changes in 39 aMCl, 50 aMCl+CeVD, 47 AD, $47 \mathrm{AD}+\mathrm{CeVD}$, and 65 healthy controls (HCs) and their associations with cognitive impairment in the executive/ attention and memory domains.

(Continued on next page)

\footnotetext{
* Correspondence: helen.zhou@duke-nus.edu.sg

${ }^{1}$ Centre for Cognitive Neuroscience, Neuroscience and Behavioural Disorders Program, Duke-National University of Singapore Medical School, Singapore, Singapore

${ }^{4}$ Clinical Imaging Research Centre, The Agency for Science, Technology and Research and National University of Singapore, Singapore, Singapore Full list of author information is available at the end of the article
}

(c) The Author(s). 2018 Open Access This article is distributed under the terms of the Creative Commons Attribution 4.0 International License (http://creativecommons.org/licenses/by/4.0/), which permits unrestricted use, distribution, and reproduction in any medium, provided you give appropriate credit to the original author(s) and the source, provide a link to the Creative Commons license, and indicate if changes were made. The Creative Commons Public Domain Dedication waiver (http://creativecommons.org/publicdomain/zero/1.0/) applies to the data made available in this article, unless otherwise stated. 
(Continued from previous page)

Results: We demonstrate divergent DMN and ECN functional connectivity changes in CeVD and non-CeVD subjects. Compared with controls, intra-DMN hippocampal functional connectivity reductions were observed in both $A D$ and $A D+C e V D$, while intra-DMN parietal and medial prefrontal-parietal functional connectivity was higher in $A D+C e V D$ and $a M C l+C e V D$, but lower in AD. Intra-ECN frontal functional connectivity increases and frontoparietal functional connectivity decreases occurred in CeVD but not non-CeVD subjects. Such functional connectivity alterations were related with cognitive impairment in a dissociative manner: intra-DMN functional connectivity changes were associated with worse cognition primarily in non-CeVD groups, while intra-ECN functional connectivity changes were associated with worse cognition primarily in CeVD groups. Additionally, CeVD and non-CeVD groups showed overlapping and distinct alterations in inter-network DMN-ECN functional connectivity depending on disease severity. In contrast to functional connectivity, CeVD groups had greater network structural connectivity damage compared with non-CeVD groups in both aMCl and AD patients. Network structural connectivity damage was associated with worse cognition.

Conclusions: We demonstrate differential functional and structural network changes between aMCI and AD patients with and without CeVD through diverging and deleterious network-based degeneration underlying domain-specific cognitive impairment.

Keywords: Neurodegeneration, Network, Functional connectivity, Structural connectivity, Alzheimer's disease, Cerebrovascular disease, Diffusion tensor imaging

\section{Background}

Alzheimer's disease (AD) with concomitant cerebrovascular disease (CeVD) is a leading cause of age-related cognitive impairment [1]. Such a mixed pathology is not only associated with distinct neurodegenerative patterns, but also with greater cognitive decline and earlier dementia onset than AD or CeVD only [2-4].

The network-based degeneration hypothesis suggests that the disease-related spread of degeneration follows a pattern based on existing brain networks [5-8]. Emerging evidence illustrates that $\mathrm{AD}$ and mild cognitive impairment (MCI) are associated with functional connectivity (FC) and structural connectivity (SC) alterations in the default mode network (DMN) with associated memory impairment, while CeVD shows FC and SC changes in the executive control network (ECN) [9-13]. Recent findings from our group using single DMN/ECN seeds indicate differential neural network changes that may be reflective of different underlying pathology in subjects with and without CeVD [14]. However, most studies have used single seed-based approaches to assess FC changes in concomitant CeVD and AD. Thus, given the multiple DMN and ECN core regions and accumulative evidence on seed-dependent FC patterns, such a region-based effect of $\mathrm{CeVD}$ on their network connectivity in $\mathrm{AD}$ and amnestic MCI (aMCI) using simultaneous FC and SC approaches remains to be elucidated $[6,7,15,16]$. Furthermore, increased vascular burden could influence cognition through network dysfunction via impaired SC [2, 17-19]. Indeed, CeVD markers have been associated with cognition in MCI [20-22]. However, the effect of CeVD on functional and structural network connectivity in $\mathrm{AD}$ needs further investigation, especially in aMCI $[22,23]$.
Given these gaps, we aimed to concurrently assess FC and SC changes within and between the DMN and ECN in aMCI and AD subjects with and without CeVD and their associations with cognitive decline using a multiple seed-based approach. We hypothesized that non-CeVD groups would show DMN FC damage underlying memory impairment while CeVD participants would show ECN FC damage underlying attention and executive function impairment. Such network divergence patterns would be less evident in SC; instead, SC disruptions are likely to be more severe in CeVD than non-CeVD.

\section{Methods}

\section{Participants}

Subjects were recruited from the following sites in Singapore: memory clinics at the National University Hospital, Singapore, St. Luke's Hospital, and the community as described previously [24]. The study was approved by the National Healthcare Group Domain-Specific Review Board and conducted in accordance with the Declaration of Helsinki. Written informed consent was obtained from all participants in their preferred language prior to the start of the study. All subjects underwent medical and demographic questionnaires, physical, extensive clinical and neuropsychological assessments, neuroimaging, and diagnosis. Diagnoses of cognitive impairment and dementia were made at weekly consensus meetings where clinical features, blood investigations, psychometrics, and neuroimaging data were reviewed [25]. Detailed diagnostic criteria for aMCI, aMCI+CeVD, $\mathrm{AD}$, and $\mathrm{AD}+\mathrm{CeVD}$, and inclusion/exclusion criteria are provided in Additional file 1. In brief, magnetic resonance imaging (MRI) scans were used to produce a visual rating 
of cortical infarcts, lacunes, and confluent white matter lesions in the brain which determined significant CeVD based on prior criteria (see Additional file 1 and our previous work [26-31]). After excluding 43 participants who either did not have MRI/diffusion tensor imaging (DTI)/ functional MRI (fMRI) scans or did not pass neuroimaging data quality control, a total of 248 participants comprising $39 \mathrm{aMCI}, 50 \mathrm{aMCI}+\mathrm{CeVD}, 47 \mathrm{AD}, 47 \mathrm{AD}+\mathrm{CeVD}$, and 65 healthy controls (HCs) were included in our study. There were no differences in disease severity or cognition between CeVD and non-CeVD groups at each AD stage (Table 1). Patients who completed cognitive assessments were included in brain cognition association analyses.

\section{Neuropsychological assessment}

Diagnoses of dementia were made at weekly consensus meetings following a review of the patient's clinical history, blood work, neuropsychological assessments, and neuroimaging data, conducted by neurologists, neuropsychologists, research nurses, and research assistants following our previous work [25, 32]. Trained research psychologists administered the following cognitive screening tests: the Mini-Mental State Examination (MMSE), the Clinical Dementia Rating Scale (CDR), the
Montreal Cognitive Assessment, informant questionnaire on cognitive decline, and a detailed neuropsychological test battery locally validated for older Singaporeans [33], which assessed the following seven domains [26]: executive function (frontal assessment battery [34]; maze task [35]), attention (digit span; visual memory span [36]; auditory detection [37]), language (Boston naming test [38]; verbal fluency [39]), visuomotor speed (symbol digit modality test [40]; digit cancellation [41]), visuoconstruction (Weschler memory scale-revised visual reproduction copy task [36]; clock drawing [42]; Weschler adult intelligence scale-revised subtest of block design [43]), verbal memory (word list recall [44]; story recall), and visual memory (picture recall; visual reproduction [36]). $Z$ scores were then derived for individual subtests and adapted such that a larger value reflects better performance. Summing the $z$ scores of each subtest and subsequently dividing by the number of the subtests under that domain computed the overall $z$ score for each individual domain. Domain-specific $z$ scores were used to compute the final global cognitive composite score. The visual and verbal memory scores were combined into a composite memory score. Only subjects who completed all the tasks were included in the statistical analysis on cognition.

Table 1 Demographics and clinical characteristics of patients and control subjects

\begin{tabular}{|c|c|c|c|c|c|c|}
\hline & $\mathrm{HC}(n=65)$ & $\mathrm{aMCl}(n=39)$ & $\mathrm{aMCl}+\mathrm{CeVD}(n=50)$ & $\mathrm{AD}(n=47)$ & $\mathrm{AD}+\mathrm{CeVD}(n=47)$ & $p$ value \\
\hline Age (years) & $67.3(6.2)^{b, c, d, e}$ & $71.8(7.9)^{d}$ & $71.4(8.7)^{d}$ & $75.2(7.8)$ & $79.3(6.1)$ & $p<0.001$ \\
\hline Gender (M/F), $n$ & $29 / 36$ & $22 / 17$ & $29 / 21$ & $17: 30$ & $15: 32$ & $p=0.037$ \\
\hline Handedness (R:L), $n$ & $60: 5$ & $37: 2$ & $50: 0$ & $47: 0$ & $47: 0$ & $p=0.019$ \\
\hline Ethnicity (C:M:I:O), n & $60 / 2 / 3 / 0$ & $34 / 1 / 4 / 0$ & $38 / 8 / 2 / 2$ & $38 / 6 / 1 / 2$ & $34 / 9 / 4 / 0$ & $p=0.016$ \\
\hline CDR global & $0.1(0.2)$ & $0.4(0.2)$ & $0.4(0.3)$ & $1.1(0.3)$ & $1.3(0.5)$ & $p<0.001$ \\
\hline CDR sum of boxes & $0.1(0.2)^{d, e}$ & $0.9(0.7)^{\mathrm{d}, \mathrm{e}}$ & $0.9(0.9)^{\mathrm{d}, \mathrm{e}}$ & $6.2(2.2)$ & $6.9(2.9)$ & $p<0.001$ \\
\hline MMSE & $27.4(2.0)^{b, c, d, e}$ & $24.4(3.5)^{\mathrm{d}, \mathrm{e}}$ & $23.6(3.6)^{\mathrm{d}, \mathrm{e}}$ & $16.6(5.2)$ & $16.4(4.4)$ & $p<0.001$ \\
\hline GDS & $1.57(2.11)^{\mathrm{e}}$ & $1.74(2.71)$ & $2.52(3.02)$ & $2.40(2.29)$ & $3.21(3.16)$ & $p=0.015$ \\
\hline \multirow[t]{2}{*}{$\mathrm{WMH}, \mathrm{cm}^{3}$} & $1.97(1.67)^{c, e}$ & $3.23(3.11)^{c, e}$ & $14.53(14.74)^{d}$ & $5.30(4.84)^{\mathrm{e}}$ & $18.11(13.42)$ & $p<0.001$ \\
\hline & $n=57$ & $n=39$ & $n=49$ & $n=37$ & $n=34$ & Overall ANOVA \\
\hline Executive & $0.81(0.40)^{b, c, d, e}$ & $0.27(0.70)^{d, e}$ & $0.01(0.71)^{d, e}$ & $-0.93(1.08)$ & $-1.07(0.98)$ & $p<0.001$ \\
\hline Attention & $0.63(0.42)^{b, c, d, e}$ & $0.11(0.56)^{d, e}$ & $-0.005(0.52)^{\mathrm{d}, \mathrm{e}}$ & $-0.69(0.91)$ & $-0.62(0.67)$ & $p<0.001$ \\
\hline Language & $0.81(0.44)^{b, c, d, e}$ & $0.14(0.55)^{d, e}$ & $0.03(0.48)^{\mathrm{d}, \mathrm{e}}$ & $-0.88(0.86)$ & $-0.97(0.70)$ & $p<0.001$ \\
\hline Verbal memory & $0.092(0.47)^{b, c, d, e}$ & $-0.13(0.64)^{\mathrm{d}, \mathrm{e}}$ & $-0.13(0.59)^{d, e}$ & $-0.86(0.51)$ & $-0.88(0.30)$ & $p<0.001$ \\
\hline Visual memory & $0.97(0.37)^{b, c, d, e}$ & $-0.12(0.49)^{d, e}$ & $-0.13(0.39)^{\mathrm{d}, \mathrm{e}}$ & $-0.92(0.50)$ & $-0.90(0.46)$ & $p<0.001$ \\
\hline Visuoconstruction & $0.83(0.37)^{b, c, d, e}$ & $0.21(0.61)^{d, e}$ & $-0.07(0.65)^{\mathrm{d}, \mathrm{e}}$ & $-0.79(0.93)$ & $-0.91(0.76)$ & $p<0.001$ \\
\hline Visuomotor & $0.85(0.47)^{b, c, d, e}$ & $0.18(0.68)^{d, e}$ & $-0.10(0.68)^{d, e}$ & $-0.85(0.74)$ & $-0.89(0.66)$ & $p<0.001$ \\
\hline
\end{tabular}

Out of the total 248 participants with imaging data, 246 participants had functional connectivity data and 247 had structural connectivity data. Out of the total 217 subjects with cognitive data, 215 had functional connectivity data and 216 had structural connectivity data

Values represent mean (SD) unless otherwise indicated

Superscript letters indicate whether group mean was significantly different compared with ${ }^{b}$ aMCl (B), ' $a M C l$ with CeVD, ${ }^{d} A l z h e i m e r ' s$ disease, and ${ }^{e}$ Alzheimer's disease with CeVD, based on post-hoc comparisons $(p<0.05)$ following one-way analysis of variance

Chi-square tests were carried out on sex and CDR global, while Fisher's exact test was carried out for handedness and ethnicity covariates

$A D$ Alzheimer's disease, $a M C I$ amnestic mild cognitive impairment, ANCOVA analysis of covariance, $C$ Chinese, CeVD cerebrovascular disease, CDR Clinical Dementia Rating, $F$ female, GDS Geriatric Depression Scale, $H C$ healthy controls, I Indian, $L$ left, $M$ Malay, $M$ male, MMSE Mini-Mental State Examination, $O$ other, $R$ right, $W M H$ white matter hyperintensity 


\section{Image acquisition}

All subjects underwent an MRI brain scan using the 3-T Tim Trio system (Siemens, Erlangen, Germany), including a 5-min task-free fMRI scan, a T1-weighted magnetization prepared rapid gradient recalled echo sequence, a fluid attenuated inversion recovery sequence, and a DTI scan using a single-shot, echo-planar imaging sequence. White matter hyperintensity (WMH) segmentation on FLAIR images was performed using an automated procedure as described in our previous work [45, 46]. Further details are provided in Additional file 1 (Supplementary Methods).

\section{Image preprocessing}

Task-free fMRI images were preprocessed using the Analysis of Functional Neuroimages software [47] and the FMRI Software Library (FSL) [48], following our previous protocol [24, 49]. Task-free fMRI preprocessing steps comprised the following: 1) discarding the first five images for signal stabilization and subject adaptation; 2) slice time and head motion correction; 3) despiking and grand mean scaling; 4) spatial smoothing with a 6-mm FWHM Gaussian kernel, temporal band pass filtering $(0.009-0.1 \mathrm{~Hz})$ and detrending (first and second order); 5) coregistering to structural MRI using boundary-based registration $(\mathrm{BBR}) ; 6)$ nonlinearly normalizing to the standard MNI space (FNIRT) via T1-weighted structural MR image; and 7) regressing out signals from white matter, cerebrospinal fluid signals, whole-brain global signal, and six head-motion parameters.

The DTI data were preprocessed by FSL following methods previously used in our studies [50]. Head movement and eddy current distortion were corrected through affine registration of diffusion-weighted images to the first $\mathrm{b}=0$ volume [51]. Data with a maximum displacement relative to the first $\mathrm{b}=0$ volume more than $3 \mathrm{~mm}$ were discarded. Diffusion gradients were rotated with reference to the motion parameters to improve data consistency. Individual maps were visually inspected for signal dropout and artifacts. To enable probabilistic tractography, the probabilistic distribution of diffusion parameters at each voxel was built up by Bayesian estimation of diffusion parameters (bedpostx) [52, 53].

\section{Network connectivity derivation \\ Region of interest (ROI) derivation}

To test whether and how the DMN and ECN structural and functional network phenotype patterns in aMCI and $\mathrm{AD}$ with and without $\mathrm{CeVD}$ vary by seeding different key network regions, we employed a multiple-seed approach. We defined nine ROIs covering the DMN and ECN regions. Each spherical ROI was created with their centers determined as key nodes in the DMN and ECN by previous studies (MarsBaR package; SC: $10 \mathrm{~mm}$ radius; FC: $6 \mathrm{~mm}$ radius) [6, 8, 54]. ECN seeds included the left and right dorsolateral prefrontal cortex (IDLPFC and rDLPFC; \pm 451645 [54]) and the left and right posterior parietal cortex (IPPC and rPPC; $\pm 50-5051$ [54]). DMN seeds included the left and right parahippocampal cortex (lParaHC and rParaHC; $\pm 22-10-24$ [8]), medial prefrontal cortex (mPFC; -164844 [6]), and medial parietal regions (posterior cingulate cortex (PCC); $-7-43$ 33 [54]; and precuneus (PCUN); 1 -60 30 [54]). Seeds involved in the network SC analyses were placed to be centered in gray matter while at the same time sized at $10 \mathrm{~mm}$ to increase their chances of touching the gray and white matter boundary following previous studies $[55,56]$.

\section{Functional connectivity $(F C)$ derivation}

Intrinsic connectivity networks using all nine seeds at the individual level were obtained using a seed-based approach following our previous work [57]. The mean time series of each spherical ROI were extracted from each participant's preprocessed functional images. Subsequently, Pearson's correlation was computed between each voxel's spontaneous BOLD (blood oxygen level-dependent signal) time series and the average time series for each ROI and converted to $z$ scores using Fisher's $r$-to- $z$ transformation. Group-averaged functional connectivity maps for each seed were calculated for each group, which demonstrated highly overlapping but distinctive connectivity in the DMN and ECN (Additional file 1: Figure S1).

\section{Structural connectivity (SC) derivation}

Each individual's diffusion image was first coregistered to the corresponding high-resolution $\mathrm{T} 1$ structural image using BBR [58]. T1 structural images were nonlinearly registered to the MNI space using FSL FNIRT. The derived transformation parameters were subsequently inversed and applied on the seeds to produce seeds in the diffusion native space.

We carried out probabilistic fiber tractography on these seeds using the DTI analysis software PANDA (Additional file 1: Figure S2). A sampling of $5000 \times n$ streamline fibers (5000 fibers per voxel) was carried out for each seed region with $n$ number of voxels. The connectivity probability from one seed region to a given seed region was thus defined as the number of fibers passing through the given seed divided by the total number $(5000 \times n)$ of sampled fibers $[52,53,59,60]$. The unidirectional connectivity probability $P_{\mathrm{ij}}$ between two seeds $i$ and $j$ was the weighted mean of the two individual connectivity probabilities $\mathrm{i} \rightarrow \mathrm{j}$ and $\mathrm{j} \rightarrow \mathrm{i}$. Subsequently a subject-level SC matrix of the connectivity probabilities for all 36 edges between nine ROIs was 
created. These probabilities were then logarithmically transformed and normalized for statistical analyses.

\section{Statistical analyses \\ Functional connectivity group differences}

Second-level analyses for each seed-based FC map were performed using Statistical Parametric Mapping (SPM12; http://www.fil.ion.ucl.ac.uk/spm/software/spm12/) software. We created an analysis of covariance (ANCOVA) model where age, sex, handedness, and ethnicity were included as covariates and groups were modeled as separate covariates. Pair-wise two-sample $t$ tests were conducted to assess group differences between each of the disease groups and $\mathrm{HCs}$ as well as head-to-head comparisons between CeVD and non-CeVD groups. To study within-network and inter-network group differences, group-averaged network masks for the DMN and ECN were defined (see Additional file 1). Results were thresholded at a height threshold of $p<0.01$ and a cluster-extent threshold of $p<0.05$.

\section{Structural connectivity group differences}

For SC, all 36 edges between nine ROIs were classified as belonging to intra-DMN, intra-ECN, or inter-network DMN-ECN (Additional file 1: Table S1). We tested the group differences in SC of the two networks by performing edge-wise ANCOVA analysis on all 36 edges with age, sex, handedness, and ethnicity as nuisance variables. Bonferroni post-hoc pair-wise analyses were also performed on edges with significant group effect in the ANCOVA model. Results are reported at a threshold of $p<0.05$ following correction for multiple comparisons across groups. Following this, multiple comparisons correction across 36 edges was conducted using FWE-correction at $p<0.0013$.

\section{Association between brain connectivity and cognition}

To assess the association between intra- and inter-network SC/FC and cognition and the influence of $\mathrm{CeVD}$ on this relationship, we ran a step-wise multiple regression model across all patients (aMCI/AD) with and without CeVD separately (IBM SPSS software, version 24.0, Chicago, IL, USA). Cognitive test $z$ scores for executive function, attention, verbal memory, and visual memory domains comprised the dependent variables since they represent the major deficits observed in subjects with $\mathrm{AD}$ and $\mathrm{CeVD}[2,4]$. We built two multiple regression models for each of the four cognitive domains (one for CeVD and one for non-CeVD) in which FC measures that showed significant group differences in $\mathrm{AD}$ compared with $\mathrm{HCs}$ or $\mathrm{AD}+\mathrm{CeVD}$ compared with $\mathrm{HCs}$ comprised the independent variables. Age, sex, handedness, years of education, and ethnicity covariates comprised the nuisance variables in the model. The same multiple regression models were also built for SC measures and cognitive $z$ scores. Beta and $p$ values are reported at $p<0.0125$, corrected for multiple comparisons across the four cognitive domains.

\section{Results \\ Intra-network and inter-network group differences in functional connectivity Intra-DMN FC group differences}

Seed-based voxel-wise FC ANCOVA analyses revealed largely posterior intra-DMN temporoparietal reductions for the IParaHC, PCC, and PCUN seeds in AD subjects relative to controls. $\mathrm{AD}$ patients had decreases in intra-DMN medial prefrontal-parietal FC but increases in anterior DMN local frontal FC (Fig. 1; Additional file 1: Table S2A). Thus, AD subjects showed lower FC in both the posterior and anterior $\mathrm{DMN}$ regions compared with controls but higher FC for only the mPFC seed. aMCI subjects did not show any intra-DMN FC alterations compared with controls.

Subjects with CeVD showed overlapping but distinct intra-DMN FC changes. Specifically, $\mathrm{AD}+\mathrm{CeVD}$ subjects also showed reductions in posterior DMN FC for the bilateral ParaHC seeds and PCC-temporal and PCUN-temporal FC. However, in contrast to non-CeVD, $\mathrm{CeVD}$ groups showed increased intra-DMN FC for the mPFC, PCC, and PCUN seeds. Specifically, local intra-DMN parietal FC exhibited increases in both $\mathrm{AD}+\mathrm{CeVD}$ and $\mathrm{aMCI}+\mathrm{CeVD}$ subjects for the $\mathrm{PCC}$ and PCUN seeds. Additionally, for the mPFC seed, $\mathrm{AD}+\mathrm{CeVD}$ and $\mathrm{aMCI}+\mathrm{CeVD}$ showed increased medial prefrontal-parietal FC (Fig. 1; Additional file 1: Figure S3 and Table S2A). Overall, increases in intra-DMN FC were predominantly observed in CeVD subjects.

\section{Intra-ECN FC group differences}

Overall, intra-ECN FC was affected to the largest extent in CeVD subjects. Compared with controls, local intra-ECN FC showed increases across all four ECN seeds (i.e., IDLPFC, rDLPFC, IPPC, and rPPC), predominantly in $\mathrm{AD}+\mathrm{CeVD}$. Specifically, $\mathrm{AD}+\mathrm{CeVD}$ subjects showed increases in local frontal FC for the IDLPFC and rDLPFC seeds and increases in local parietal FC for the IPPC and rPPC seeds. On the other hand, aMCI+CeVD subjects showed both increases and decreases in frontal FC for the rDLPFC seed. No other DLPFC-related FC reductions were observed. Such increases in frontal FC for the DLPFC seeds were associated with higher WMH volume (Additional file 1: Supplementary Results 2.5 and Figure S5). Additionally, $\mathrm{AD}+\mathrm{CeVD}$ and $\mathrm{aMCI}+\mathrm{CeVD}$ showed decreased frontoparietal FC for the IPPC seed while $\mathrm{AD}+\mathrm{CeVD}$ subjects showed decreased frontal FC for the rPPC seed (Fig. 2; Additional file 1: Figure S4 and Table S2B). 


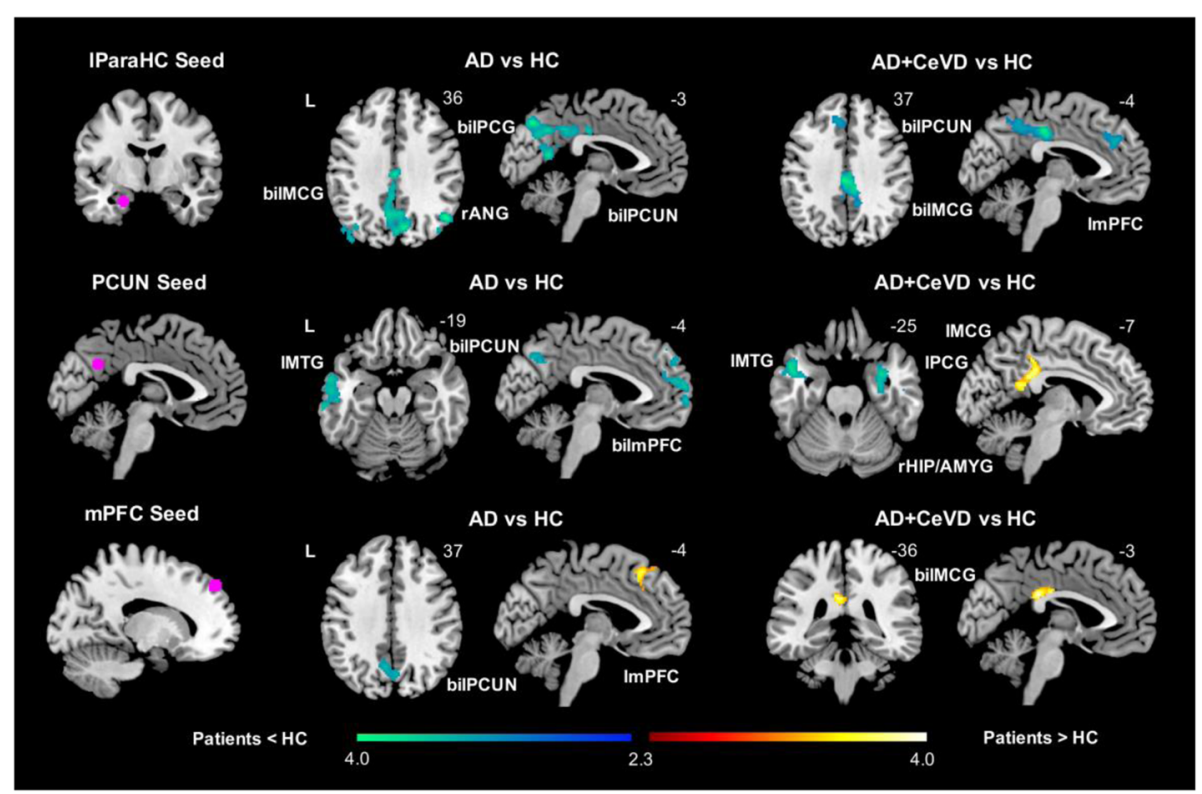

Fig. 1 Distinct default mode network functional connectivity changes in subjects with and without cerebrovascular disease. Group functional connectivity difference maps were overlaid on the MNI template brain. The maps highlight regions showing increased (hot color) or decreased functional connectivity (cold color) in patient groups compared with $\mathrm{HCs}$ for the default mode network (DMN). Both $A D$ and $A D+C e V D$ subjects had reduced intra-DMN FC in hippocampal regions of the network for the IParaHC and PCUN seeds. Some medial prefrontal-parietal/temporal and parietal FC decreases were observed in AD subjects for the IParaHC, PCUN, and MPFC seeds, while parietal FC increases were observed in $A D+C e V D$ subjects for the PCUN and mPFC seeds. AD subjects also showed increases in local medial prefrontal FC for the mPFC seed. AD Alzheimer's disease, aMCl amnestic mild cognitive impairment, ANG right angular gyrus, bil bilateral, CeVD cerebrovascular disease, FC functional connectivity, HC healthy controls, HIP/AMYG hippocampus/amygdala, I left, MCG bilateral middle cingulate gyrus, MTG left middle temporal gyrus, mPFC medial prefrontal cortex, ParaHC parahippocampal cortex, PCG bilateral posterior cingulate gyrus, PCUN precuneus, r right

In contrast, $\mathrm{AD}$ subjects did not show any intra-ECN DLPFC-related FC changes. However, for both the IPPC and rPPC seeds, AD subjects showed decreased frontoparietal FC and increased local parietal FC for the IPPC seed (Fig. 2; Additional file 1: Table S2B). Similar to intra-DMN, no intra-ECN FC alterations were observed in aMCI subjects relative to controls.

\section{Inter-network FC group differences}

Inter-network frontotemporal FC and frontoparietal FC was reduced in $\mathrm{AD}$ subjects. In contrast, $\mathrm{AD}+\mathrm{CeVD}$ subjects showed reductions in frontotemporal, temporoparietal, and frontoparietal FC, but also increases in medial prefrontal-frontal, parietal, and frontoparietal FC (Additional file 1: Table S2C). Further details are provided in Additional file 1 (Supplementary Results).

\section{Group differences in intra- and inter-network FC between $\mathrm{CeVD}$ and non-CeVD groups}

Head-to-head comparisons between CeVD and non-CeVD groups were consistent with intra-DMN and intra-ECN FC changes compared with controls and are detailed in Additional file 1 (Supplementary Results and Table S3). Inter-network DMN-ECN FC showed disease stage-dependent divergent alterations between CeVD and non-CeVD subjects (Additional file 1: Table S3C). Further details are provided in Additional file 1 (Supplementary Results).

Although none of our participants had a clinical diagnosis of depression, to control for the potential influence of mild depressive symptoms on FC we repeated the same analyses on group differences in intra- and inter-network FC after controlling for Geriatric Depression Scale scores. All the main findings remained the same (Additional file 1: Tables S2 and S3).

\section{CeVD groups had more early and severe structural connectivity disruptions than non-CeVD groups}

Intra- and inter-network SC progressively worsened from controls to aMCI and AD (Additional file 1: Table S3). The largest reduction in SC was observed in $\mathrm{AD}$ $+\mathrm{CeVD}$ subjects. Both $\mathrm{AD}$ subjects with and without CeVD showed intra-DMN temporal, frontoparietal, and temporoparietal (Fig. 3a), intra-ECN frontal, frontoparietal, and parietal (Fig. 3b), and inter-network frontal, parietal, frontoparietal, and temporoparietal SC disruptions (Fig. 3c). However, SC reductions were more widespread in $\mathrm{CeVD}$ than in non-CeVD groups.

At the aMCI stage, aMCI subjects without $\mathrm{CeVD}$ showed no $\mathrm{SC}$ changes. In contrast, $\mathrm{aMCI}+\mathrm{CeVD}$ 


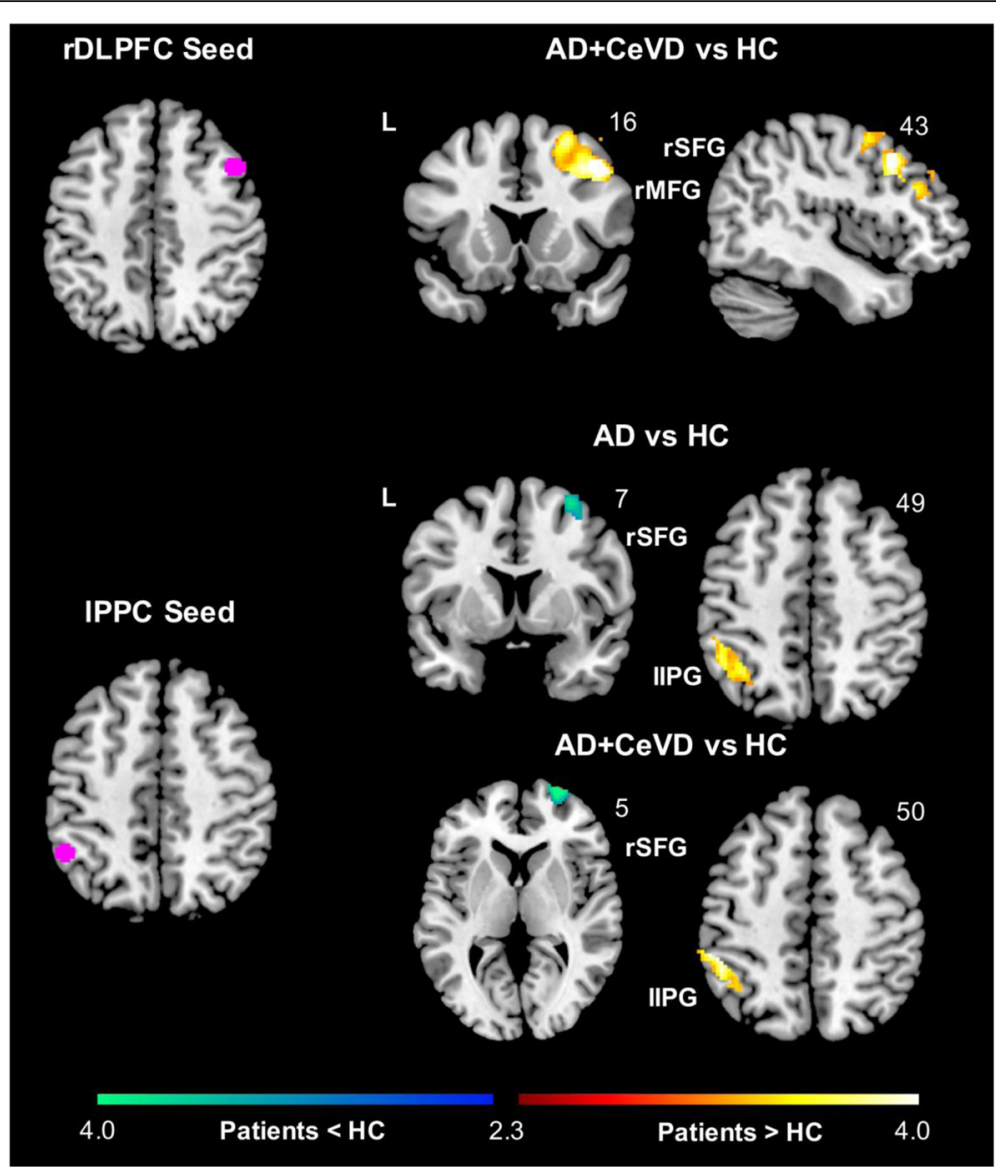

Fig. 2 Distinct executive control network functional connectivity changes in subjects with and without cerebrovascular disease. Group functional connectivity difference maps were overlaid on the MNI template brain. The maps highlight regions showing increased (hot color) or decreased functional connectivity (cold color) in patient groups compared with HCs for the executive control network (ECN). AD+CeVD subjects showed increased intra-ECN frontal FC for the rDLPFC seed and parietal FC for the IPPC seed while AD subjects showed higher parietal FC compared with HCs for the IPPC seed. Frontoparietal FC was reduced in AD and AD+CeVD subjects compared with HCs for the IPPC seed. AD Alzheimer's disease, aMCl amnestic mild cognitive impairment, ANG right angular gyrus, CeVD cerebrovascular disease, FC functional connectivity, $\mathrm{HC}$ healthy controls, IPG inferior parietal gyrus, I left, IPPC left posterior parietal cortex, MFG middle frontal gyrus, rDLPFC right dorsolateral prefrontal cortex, r right, SFG superior frontal gyrus

participants had intra-ECN (rDLPFC-IPPC; IDLPFCrDLPFC) and ECN-DMN (rDLPFC-mPFC; rDLPFC-PCC) SC reductions compared with controls (Fig. 3; Additional file 1: Table S3). Frontoparietal intra-ECN (rDLPFC-IPPC; IDLPFC-rDLPFC) and inter-network DMN-ECN (rDLPFC-PCC; IDLPFC-PCC) connections also showed reduced $\mathrm{SC}$ in $\mathrm{aMCI}+\mathrm{CeVD}$ and $\mathrm{AD}+\mathrm{CeVD}$ subjects compared with non-CeVD aMCI and AD subjects, respectively (Table 2). Such a pattern of SC deterioration remained after controlling for log-transformed WMH volume (Additional file 1: Table S4).

CeVD and non-CeVD groups feature differential structural and functional dysconnectivity underlying memory and executive functioning deficits

Intra-DMN FC associations with cognition in both memory and non-memory domains were primarily observed in $\mathrm{AD}$ and aMCI subjects without $\mathrm{CeVD}$ (Additional file 1: Table S5). Specifically, higher frontal intra-DMN FC (mPFC-left superior medial frontal gyrus) was associated with worse executive $(p=0.003 ; \quad r=0.28)$, attention $(p=0.003 ; \quad r=0.31)$ (Fig. 4a), and visual memory $(p=0.007 ; r=0.28)$. Furthermore, higher temporoparietal (PCC-lParaHC; lParaHC-bilateral precuneus) $\mathrm{FC}$ was associated with better executive function $(p=0.009 ; r=0.36)$ and visual memory $(p=0.006 ; r=0.30)$ (Fig. 4c), respectively, and higher parietal (PCC-right angular gyrus) FC was associated with better verbal memory $(p=$ $0.002 ; r=0.28$ ) (Fig. 4b). In $\mathrm{AD}+\mathrm{CeVD}$ and aMCI $+\mathrm{CeVD}$ subjects, only higher temporoparietal (lParaHC-bilateral middle cingulum) $\mathrm{FC}$ was associated with better verbal $(p=0.005 ; r=0.30)$ and visual memory ( $p=0.002 ; r=0.30)$ (Fig. $4 \mathrm{~d}$ ). 

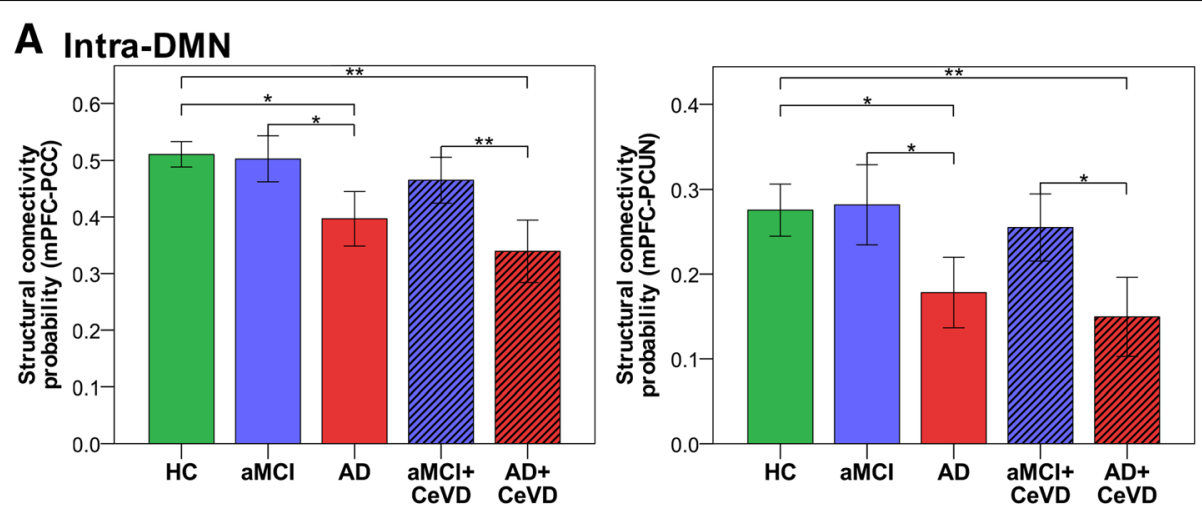

\section{B Intra-ECN}
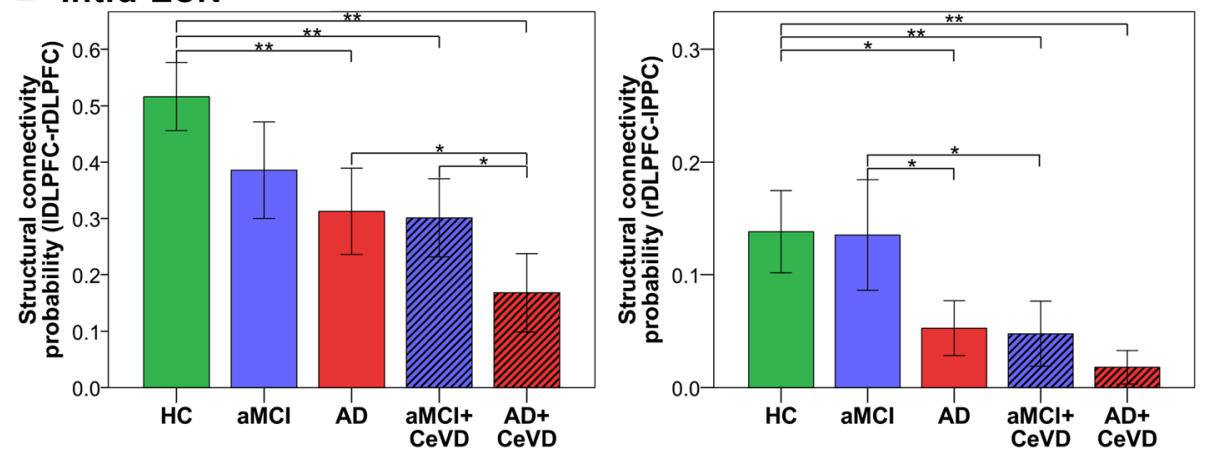

\section{DMN-ECN}
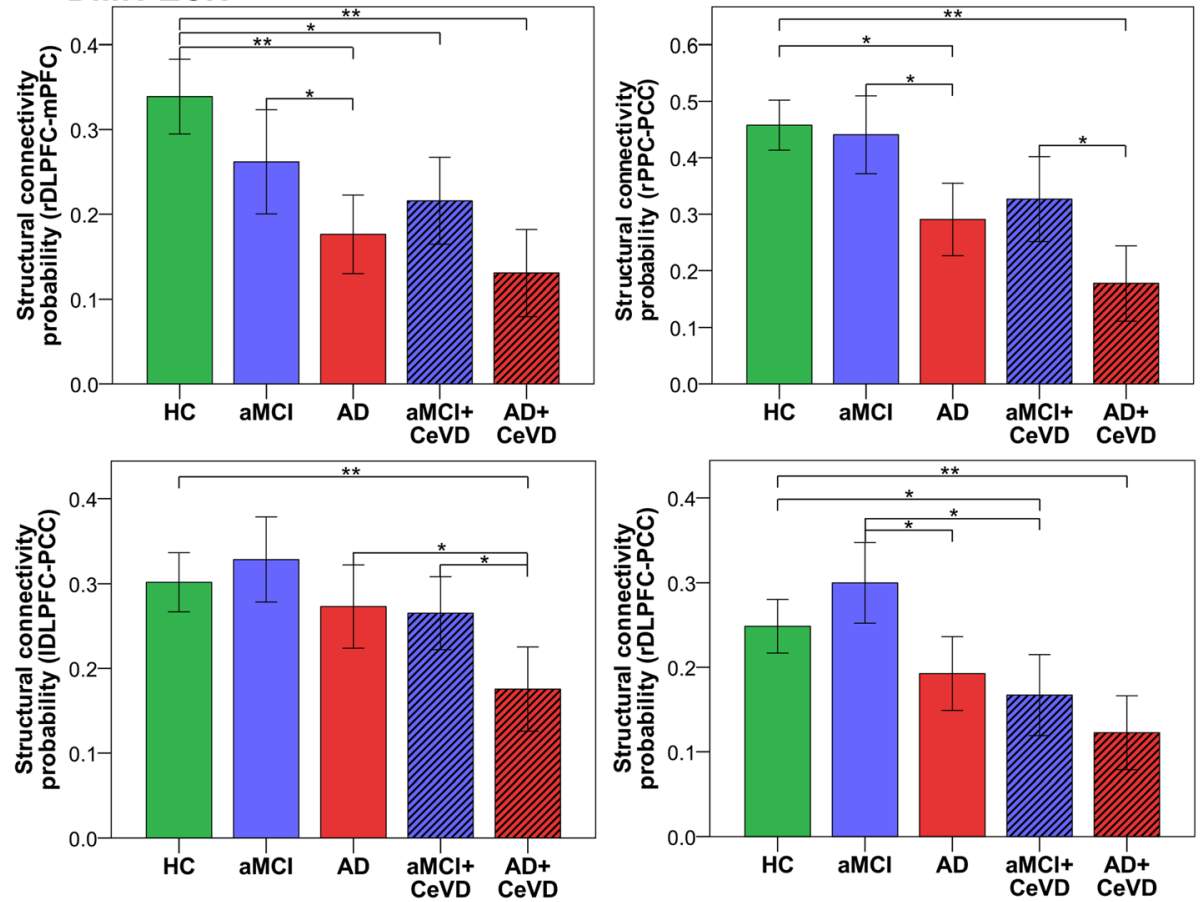

Fig. 3 (See legend on next page.) 
(See figure on previous page.)

Fig. 3 Patients with cerebrovascular disease had more severe and early structural connectivity disruptions. Representative edges showing group differences in structural connectivity. a Both $A D$ and $A D+C e V D$ subjects had reduced intra-DMN SC compared with both controls and aMCl $+\mathrm{CeVD}$ but AD+CeVD subjects had more widespread damage (Table 2). $\mathbf{b}$ Intra-ECN SC was reduced in AD+CeVD compared with controls and $\mathrm{aMCl}+\mathrm{CeVD}$ as well as AD participants (left). Additionally, subjects with aMCl+CeVD also had reduced intra-ECN SC compared with controls and aMCl only subjects (right). $\mathbf{c}$ Inter-network SC showed reduced connectivity in AD+CeVD compared with controls and aMCl+CeVD and in AD subjects compared with controls and aMCl. Additionally, the bottom two panels illustrate SC disruption between AD+CeVD and AD as well as $\mathrm{aMCl}+\mathrm{CeVD}$ and $\mathrm{aMCl}$ participants. Pairwise comparisons were corrected for multiple comparisons. ${ }^{*} p<0.05,{ }^{* *} p<0.001$. AD Alzheimer's disease, aMCI amnestic mild cognitive impairment, CeVD cerebrovascular disease, DMN default mode network, ECN executive control network, HC healthy controls, IDLFPC left dorsolateral prefrontal cortex, IPPC left posterior parietal cortex, mPFC medial prefrontal cortex, PCC posterior cingulate cortex, PCUN precuneus, rDLFPC right dorsolateral prefrontal cortex, rPPC right posterior parietal cortex, SC structural connectivity

Table 2 Group differences in structural connectivity

\begin{tabular}{|c|c|c|c|c|c|c|c|c|}
\hline & \multicolumn{3}{|c|}{ Without CeVD } & \multicolumn{3}{|l|}{ With CeVD } & \multirow[b]{2}{*}{$\begin{array}{l}\mathrm{aMCl}>\mathrm{aMCl} \\
+\mathrm{CeVD}\end{array}$} & \multirow[b]{2}{*}{$\begin{array}{l}A D>A D \\
+C e V D\end{array}$} \\
\hline & $\mathrm{HC}>\mathrm{aMCl}$ & $\mathrm{HC}>\mathrm{AD}$ & $\mathrm{aMCl}>\mathrm{AD}$ & $\begin{array}{l}\mathrm{HC}>\mathrm{aMCl} \\
+\mathrm{CeVD}\end{array}$ & $\begin{array}{l}\mathrm{HC}>\mathrm{AD} \\
+\mathrm{CeVD} \\
\end{array}$ & $\begin{array}{l}\mathrm{aMCl}+\mathrm{CeVD}>\mathrm{AD} \\
+\mathrm{CeVD}\end{array}$ & & \\
\hline \multicolumn{9}{|l|}{ Intra-DMN } \\
\hline $\begin{array}{l}\text { IParaHC- } \\
\text { rParaHC }\end{array}$ & - & - & 0.019 & - & 0.032 & - & - & - \\
\hline IParaHC-mPFC & - & - & - & - & 0.002 & - & - & - \\
\hline IParaHC-PCUN & - & - & - & - & - & 0.010 & - & - \\
\hline rParaHC-PCC & - & - & - & - & 0.030 & 0.019 & - & - \\
\hline mPFC-PCC & - & 0.002 & 0.041 & - & $<0.001^{*}$ & $<0.001^{*}$ & - & - \\
\hline mPFC-PCUN & - & 0.003 & 0.011 & - & $<0.001^{*}$ & 0.003 & - & - \\
\hline \multicolumn{9}{|l|}{ Intra-ECN } \\
\hline IDLPFC-rDLPFC & - & $0.001^{*}$ & - & $0.001^{*}$ & $<0.001^{*}$ & 0.025 & - & 0.039 \\
\hline IDLPFC-rPPC & - & $<0.001^{*}$ & 0.042 & - & $<0.001^{*}$ & - & 0.004 & - \\
\hline rDLPFC-IPPC & - & 0.004 & 0.008 & $0.001^{*}$ & $<0.001^{*}$ & - & - & - \\
\hline rDLPFC-rPPC & - & - & - & - & 0.027 & - & - & - \\
\hline IPPC-rPPC & - & $0.001^{*}$ & 0.010 & - & $0.001^{*}$ & - & - & - \\
\hline \multicolumn{9}{|l|}{ DMN-ECN } \\
\hline IDLPFC-IParaHC & - & - & - & - & $0.001^{*}$ & - & - & - \\
\hline IDLPFC-PCC & - & - & - & - & $<0.001^{*}$ & 0.008 & - & 0.007 \\
\hline IDLPFC-PCUN & - & - & - & - & 0.021 & - & - & - \\
\hline rDLPFC-mPFC & - & $<0.001^{*}$ & 0.028 & 0.004 & $<0.001^{*}$ & - & - & - \\
\hline rDLPFC-PCC & - & - & 0.004 & 0.037 & $<0.001^{*}$ & - & $0.001^{*}$ & - \\
\hline rDLPFC-PCUN & - & - & - & - & 0.007 & - & - & - \\
\hline IPPC-rParaHC & - & - & 0.022 & - & 0.047 & - & - & - \\
\hline IPPC-mPFC & - & - & - & - & - & 0.043 & - & - \\
\hline IPPC-PCC & - & - & - & - & $<0.001^{*}$ & - & - & - \\
\hline rPPC-rParaHC & - & - & - & - & $0.001^{*}$ & 0.023 & - & - \\
\hline $\mathrm{rPPC}-\mathrm{mPFC}$ & - & - & - & - & $<0.001^{*}$ & - & - & - \\
\hline rPPC-PCC & - & 0.021 & 0.010 & - & $<0.001^{*}$ & 0.023 & - & - \\
\hline
\end{tabular}

Structural connectivity probabilities were logarithmically transformed and normalized for statistical analyses. There were no group differences in SC between $\mathrm{HCS}$ and aMCI without CeVD subjects. AD+CeVD subjects had the largest reduction in SC at both the intra- and inter-network level compared with both $\mathrm{HCS}$ and aMCI with and without CeVD. We detected a reduction in $\mathrm{SC}$ in aMCl+CeVD subjects compared with both controls and aMCl subjects. $\mathrm{AD}+\mathrm{CeVD}$ and aMCl+CeVD showed intra-ECN and inter-network SC reductions compared with $A D$ and aMCI without CeVD subjects, respectively

Each cell represents the $p$ value for significant pair-wise comparisons in $\mathrm{SC}$ at a threshold of $p<0.05$ following correction for multiple comparisons across groups $A D$ Alzheimer's disease, $a M C l$ amnestic mild cognitive impairment, $C e V D$ cerebrovascular disease, $D M N$ default mode network, ECN executive control network, HC healthy controls, IDLFPC left dorsolateral prefrontal cortex, IParaHC left parahippocampus, IPCC left posterior cingulate cortex, IPPC left posterior parietal cortex, mPFC medial prefrontal cortex, $r D L F P C$ right dorsolateral prefrontal cortex, $r$ ParaHC right parahippocampus, $r P C U N$ right precuneus, $r P P C$ right posterior parietal cortex, SC structural connectivity

${ }^{*}$ Comparisons that passed multiple comparisons correction across the 36 edges at $p<0.0013$ 

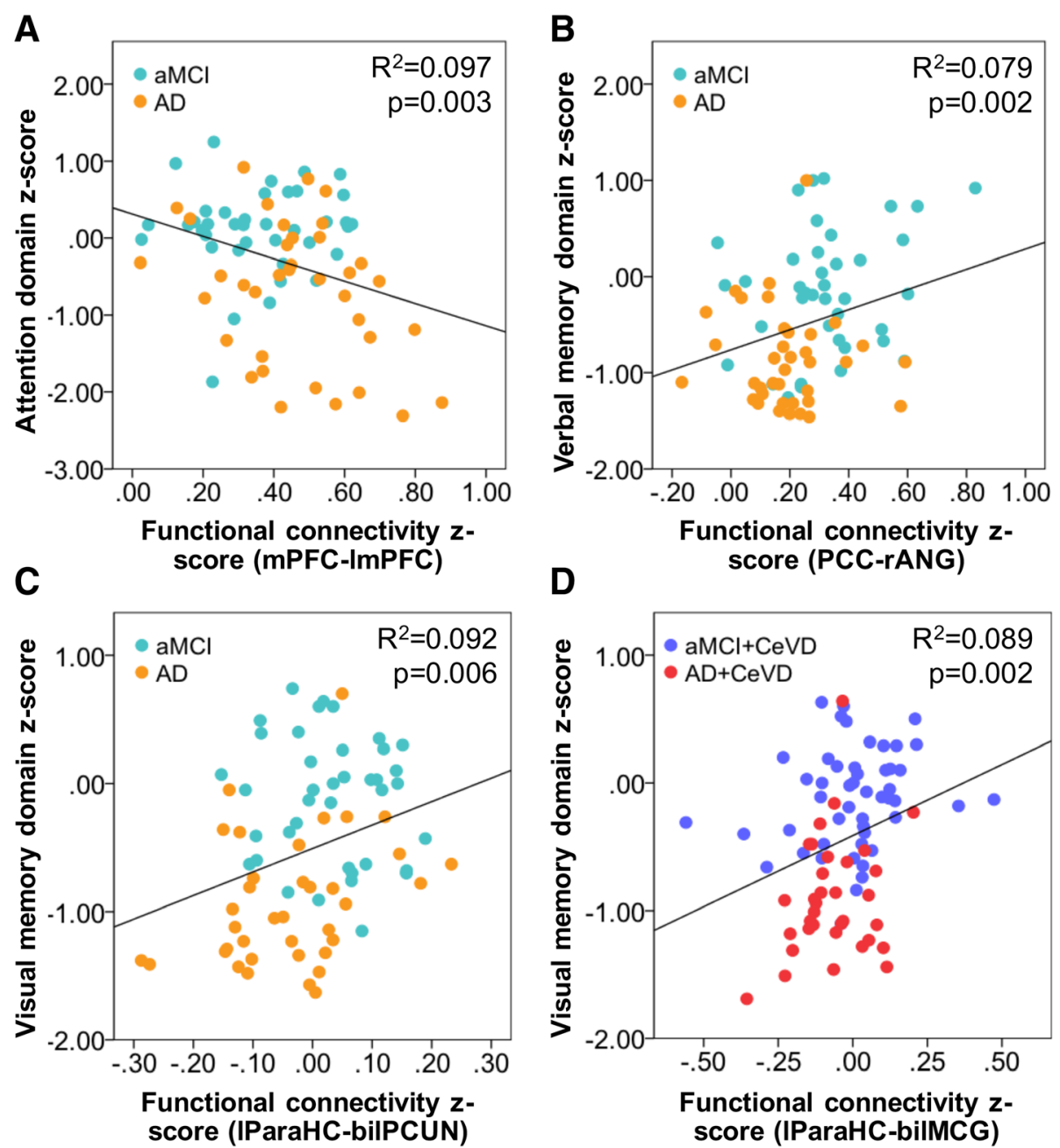

Fig. 4 Intra-DMN functional connectivity relates to memory and executive/attention function in subjects without cerebrovascular disease and memory in subjects with cerebrovascular disease. Representative regions showing associations between intra-DMN functional connectivity and cognition. In aMCI and AD subjects without CeVD, a FC between MPFC and left superior mid frontal gyrus was negatively associated attention function while $\mathbf{b}$ higher parietal FC between PCC and right angular gyrus was associated with higher verbal memory and $\mathbf{c}$ higher temporoparietal FC between IParaHC and bilateral PCUN was associated with better visual memory. In AD and aMCI subjects with CeVD, d higher temporoparietal FC between the IParaHC and bilateral middle cingulate gyrus was positively associated with visual memory. All FC cognitions shown pass the multiple comparisons correction for number of cognitive domains at $p<0.0125$. AD Alzheimer's disease, aMCI amnestic mild cognitive impairment, ANG right angular gyrus, bil bilateral, CeVD cerebrovascular disease, DLFPC dorsolateral prefrontal cortex, DMN default mode network, FC functional connectivity, I left, MCG middle cingulate gyrus, mPFC medial prefrontal cortex, ParaHC parahippocampal cortex, PCC posterior cingulate cortex, PCUN precuneus, r right, SC structural connectivity

In contrast, intra-ECN FC associations with cognition in both memory and non-memory domains were primarily observed in $\mathrm{AD}$ and aMCI subjects with CeVD (Additional file 1: Table S5). Specifically, higher frontal (IDLPFC-right middle frontal gyrus) FC was associated with worse executive function $(p=0.002 ; r=0.35)$ (Fig. 5b), attention $(p=0.003 ; r=0.33)$, and visual memory $(p=0.002 ; \quad r=0.34)$. Higher parietal FC (rPPC-left inferior parietal gyrus) was associated with better attention $(p=0.001 ; r=0.29) \quad$ (Fig. 5c) while higher frontoparietal (IPPC-right superior frontal gyrus) FC was associated with better visual memory $(p=0.006 ; r=0.31)$ (Fig. 5d). Only higher frontoparietal (rPPC-right middle superior frontal gyrus) FC was associated with better verbal memory $(p=0.005 ; r=0.36)$ in $\mathrm{AD}$ and aMCI subjects without CeVD (Fig. 5a). Additionally, there were no associations between intra-DMN FC and non-memory function in CeVD groups and between intra-ECN FC and non-memory function in non-CeVD groups.

SC deterioration was associated with cognitive impairment in a similar manner between CeVD and non-CeVD groups (Additional file 1: Table S6). These findings remained significant after controlling for total WMH 

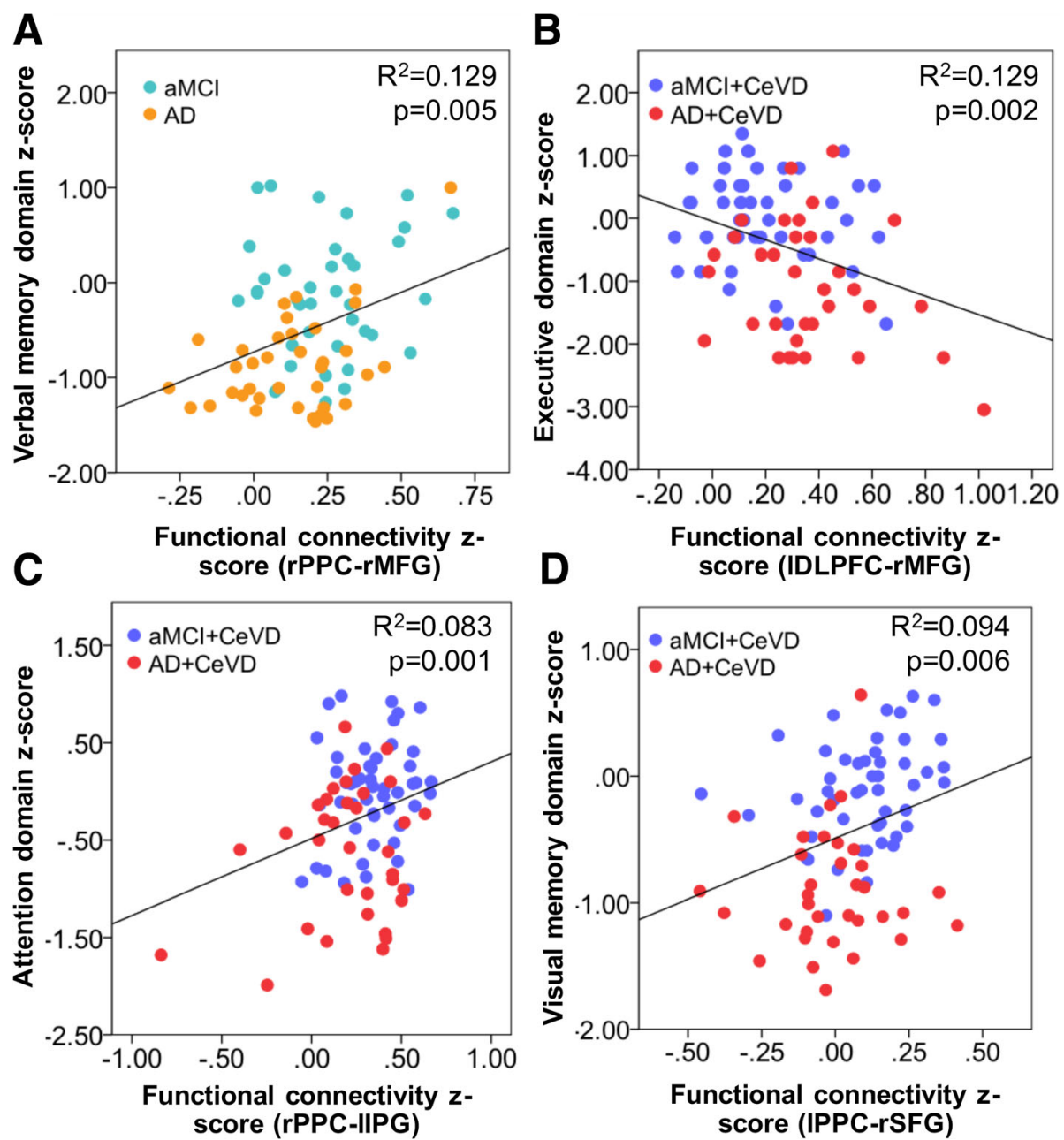

Fig. $\mathbf{5}$ Intra-ECN functional connectivity relates to memory and executive/attention in subjects with cerebrovascular disease and to memory in subjects without cerebrovascular disease. Representative regions showing associations between intra-ECN functional connectivity and cognition. a In $\mathrm{AD}$ and $\mathrm{aMCl}$ subjects without CeVD, higher frontoparietal FC between $\mathrm{rPPC}$ and right middle superior frontal gyrus was associated with better verbal memory. In $\mathrm{AD}$ and $\mathrm{aMCl}$ subjects with CeVD, $\mathbf{b}$ higher frontal FC between IDLPFC and right middle frontal gyrus was associated with worse executive function, $\mathbf{c}$ higher parietal FC between rPPC and left inferior parietal gyrus was associated with better attention function, and $\mathbf{d}$ higher frontoparietal FC between IPPC and right superior frontal gyrus was associated with better visual memory. All FC cognitions shown pass the multiple comparisons correction for number of cognitive domains at $p<0.0125$. AD Alzheimer's disease, aMCl amnestic mild cognitive impairment, CeVD cerebrovascular disease, DLPFC dorsolateral prefrontal cortex, DMN default mode network, ECN executive control network, FC functional connectivity, IPG inferior parietal gyrus, I left, MFG middle frontal gyrus, PPC posterior parietal cortex, r right, SFG superior frontal gyrus,

volume. Further details are provided in Additional file 1 (Supplementary Results).

\section{Discussion}

A hypothesis-driven multiple seed-based approach and combination of functional and structural connectivity analyses were used to assess the effect of $\mathrm{CeVD}$ on DMN and ECN connectivity in aMCI and AD patients. We demonstrated region-specific FC changes in AD patients with and without $\mathrm{CeVD}$, which related to cognitive impairment. Both $\mathrm{AD}$ and $\mathrm{AD}+\mathrm{CeVD}$ subjects showed reductions in hippocampal FC within the DMN. However, parietal and medial prefrontal-parietal DMN
FC was increased in CeVD groups but decreased in $\mathrm{AD}$ subjects. As predicted, intra-ECN alterations in frontal and frontoparietal FC were observed most extensively in CeVD subjects. Notably, aMCI+CeVD subjects exhibited similar intra-network $\mathrm{FC}$ changes to $\mathrm{AD}+\mathrm{CeVD}$, while aMCI subjects did not show any intra-network FC changes compared with HCs. Inter-network FC reductions were observed in $\mathrm{AD}$ and $\mathrm{AD}+\mathrm{CeVD}$ subjects, while aMCI and aMCI+CeVD subjects primarily showed increases when compared with controls. Direct comparisons between CeVD and non-CeVD groups revealed disease severity-dependent alterations in inter-network FC with decreased DMN-ECN FC in aMCI+CeVD 
compared with aMCI but increased DMN-ECN FC in $\mathrm{AD}+\mathrm{CeVD}$ compared with $\mathrm{AD}$. Moreover, intra-DMN FC changes were associated with cognitive impairment primarily in non-CeVD groups while ECN-related FC changes were associated with cognitive impairment primarily in CeVD groups. Additionally, CeVD groups had greater SC damage within and between the two networks compared with non-CeVD groups at both aMCI and AD stages. Similar to our FC findings, aMCI with CeVD but not those without CeVD had SC declines. This study suggests that subjects with CeVD show distinct network FC phenotypes and severe SC deterioration in the brain which underlie cognitive impairment.

The DMN is important for cognitive functions such as episodic memory and has been widely implicated in $\mathrm{AD}$ $[5,7]$. Our non-CeVD and CeVD AD patients showed extensive intra-DMN FC alterations. However, posterior DMN FC alterations involving the posterior cingulate, precuneus, and hippocampus seeds were dominant in AD subjects, as observed previously [12, 61]. These regions have been shown to comprise the core DMN as well as being involved in early amyloid deposition and associations with autobiographical and episodic memory $[5,62]$. In support of such findings, intra-DMN FC and cognition associations were primarily observed in non-CeVD groups in our study. Additionally, AD subjects showed increases in frontal FC which were negatively associated with cognition, thus indicating that such increases were derogatory in nature $[63,64]$. On the other hand, intra-DMN medial prefrontal-parietal FC was decreased in AD subjects but increased in both aMCI and AD with CeVD. Such a divergence in FC changes between CeVD and non-CeVD subjects could possibly be due to disruption of frontal pathways in the presence of vascular disease [65]. Indeed, associations between intra-ECN frontal FC increase and frontal SC decrease were found in AD groups in our study (Additional file 1: Supplementary Results section 2.6). Thus, while AD subjects both with and without CeVD showed similar involvement of hippocampal FC, medial prefrontal-parietal FC was instead differentially targeted in CeVD and non-CeVD, likely indicative of differential subnetwork FC alterations in the presence of CeVD.

Widespread intra-ECN FC alterations including increases in frontal FC were observed in $\mathrm{AD}+\mathrm{CeVD}$ subjects, possibly reflecting greater influences on ECN connectivity in CeVD [11, 14]. We also found associations between higher frontal ECN FC and higher WMH volume in both aMCI and AD groups (with and without $\mathrm{CeVD}$ ) and postulate that such increases in ECN FC could be representative of $\mathrm{CeVD}$ abnormalities in the brain (Additional file 1: Figure S5). Additionally, such increases in frontal FC were associated with worse executive, attention, and memory function in subjects with
CeVD, indicating a derogatory influence. Parietal ECN FC was reduced in CeVD subjects and was associated with worse attention function. Indeed, associations between markers of CeVD (WMH and lacunes) and executive/attention function have been demonstrated [2, 11, 18]. Moreover, task-based fMRI studies in the healthy elderly with CeVD and resting-state fMRI studies in vascular cognitive impairment have shown alterations in ECN connectivity [66]. Importantly, associations between ECN FC and cognition were primarily observed in subjects with CeVD in our study. Thus, in line with previous studies, our findings further lend evidence to the influence of concomitant $\mathrm{AD}$ and CeVD on network FC and cognition.

Furthermore, findings from our group and others show inter-network segregation as being consistently affected in $\mathrm{AD}$ patients and point towards its role in cognition [49, 67]. Interestingly, we observed lower DMN-ECN frontoparietal FC in aMCI+CeVD compared with aMCI, but higher frontoparietal $\mathrm{FC}$ in $\mathrm{AD}+\mathrm{CeVD}$ compared with AD subjects. Such differential inter-network $\mathrm{FC}$ changes at the aMCI and $\mathrm{AD}$ stages likely provide some evidence for stage-dependent alterations in network segregation in the presence of CeVD. While reductions in aMCI+CeVD inter-network $\mathrm{FC}$ possibly reflect a compensatory mechanism in the presence of CeVD, increased inter-network FC with disease progression to $\mathrm{AD}+\mathrm{CeVD}$ might reflect a breakdown in inter-network segregation possibly due to CeVD-related neuronal loss and degradation of white matter networks [68].

Prior FC studies have demonstrated inconsistent findings regarding disruptions in MCI [12, 21]. For example, whole-brain FC studies have shown both FC decreases and increases in parietal and temporal regions, reflecting a concurrent state of impairment as well as compensation [61, 63]. In this study, intra-network FC alterations were observed in aMCI+CeVD subjects when compared with controls, which largely mirrored alterations observed in $\mathrm{AD}+\mathrm{CeVD}$ subjects [14]. Interestingly, no intra-network FC alterations occurred in the aMCI-only subjects. This indicates that aMCI+CeVD subjects appear to be further along the disease spectrum than non-CeVD aMCI subjects. We speculate that the absence of FC changes might also reflect a possible compensatory mechanism accompanied by network reorganization in aMCI, which may breakdown in the presence of CeVD $[61,63]$. Further studies integrating task-based and task-free FC methods are required to study how CeVD influences whole-brain network topology and its relationship with cognitive impairment in aMCI.

In concordance with our FC patterns of large-scale alterations, our findings indicated that, overall, CeVD groups showed more widespread SC changes compared with non-CeVD groups [17]. Importantly, SC disruptions 
in $\mathrm{CeVD}$ groups occurred primarily along intra-ECN (i.e., frontal or fronto-parietal connections such as between the DLPFC and PPC). Our observations are supported by prior studies showing decreased frontal and parietal nodal efficiency in CeVD and its mediating effect on frontal lobe structure and cognition [13]. Direct comparisons between aMCI and $\mathrm{AD}$ subjects with and without CeVD also highlight greater intra-ECN SC damage. Crucially, we found early intra-ECN and inter-network SC damage with sparing of intra-DMN fibers in aMCI+CeVD subjects, in agreement with prior studies [13, 17]. As observed in our seed-based FC analyses, these differences in $\mathrm{SC}$ were not observed in the non-CeVD aMCI subjects. Such findings indicate an ECN-specific structural and additive influence of CeVD that likely begins in aMCI. Additionally, and unlike FC, there was no dissociation in the $\mathrm{SC}$-cognition relationship between CeVD and non-CeVD groups. Performance on both memory and executive/attention domains was associated with intra-network SC in both $\mathrm{AD}$ and aMCI subjects with and without CeVD, indicating that white matter damage might lead to deficiencies in both memory and executive/attention domains regardless of CeVD status [20,21]. Our SC findings reflect that CeVD may be associated with greater white matter degeneration and lend evidence to the additive hypothesis regarding the influence of $\mathrm{CeVD}$ when there is concomitant $\mathrm{AD}[3,19]$.

Our study has some limitations. As a hypothesis-driven seed-based approach was chosen to compare SC and FC in the two networks of interest, these findings may be affected by inter-subject anatomical variability. A relatively large proportion of the CeVD subjects in our study had infarcts in the frontal regions (Additional file 1: Table S8), which may bias the ECN functional connectivity estimation and associations with cognition. Additionally, although groups were not age-matched and disease duration was not available, age differences were accounted for in all analyses and disease severity was matched between $\mathrm{CeVD}$ and non-CeVD groups at the $\mathrm{aMCI}$ and $\mathrm{AD}$ stages, respectively. It has also been suggested that probabilistic fiber tracking can be influenced by the presence of WMH in the brain [69]. While we did control for WMH volume in our structural connectivity statistical analyses, WMH may still confound the fiber tracking results, especially in the crossing-fiber regions. Furthermore, out of the 248 subjects included in our study, only 45 (9 $\mathrm{HC}, 16 \mathrm{aMCI}, 14 \mathrm{aMCI}+\mathrm{CeVD}$, and $6 \mathrm{AD}$ ) had amyloid imaging data. Thus, we are unable to assess how the heterogeneity in the etiology of the patient groups, especially at the aMCI stage, would have influenced our findings.

\section{Conclusions}

In summary, we demonstrate distinct network FC phenotypes underlying cognitive impairment in patients with and without $\mathrm{CeVD}$ and provide important evidence for the influence of CeVD on early structural network disruptions. Our findings highlight the value of concurrent SC and FC neuroimaging assays to reveal early changes and distinct pathology in mixed cerebrovascular and neurodegenerative disorders. Future longitudinal studies are required to investigate the influence of CeVD on disease progression trajectory and network changes in preclinical $\mathrm{AD}$.

\section{Additional file}

Additional file 1: Supplementary methods, results, tables, and figures. (DOCX $37000 \mathrm{~kb})$

\begin{abstract}
Abbreviations
AD: Alzheimer's disease; aMCl: Amnestic mild cognitive impairment; ANCOVA: Analysis of covariance; BBR: Boundary-based registration; CeVD: Cerebrovascular disease; DMN: Default mode network; DTI: Diffusion tensor imaging; ECN: Executive control network; FC: Functional connectivity; fMRI: Functional magnetic resonance imaging; FSL: FMRI Software Library; HC: Healthy controls; IDLPFC: Left dorsolateral prefrontal cortex; IParaHC: Left parahippocampal cortex; IPPC: Left posterior parietal cortex; MCl: Mild cognitive impairment; mPFC: Medial prefrontal cortex; MRI: Magnetic resonance imaging; PCC: Posterior cingulate cortex; PCUN: Precuneus; rDLPFC: Right dorsolateral prefrontal cortex; ROI: Region of interest;

rParaHC: Right parahippocampal cortex; rPPC: Right posterior parietal cortex; SC: Structural connectivity; WMH: White matter hyperintensity

Funding

This research was supported by an NMRC Centre Grant (NMRC/CG/013/ 2013 and NMRC/CG/NUHS/2010 to (LHC), the Biomedical Research Council, Singapore (BMRC 04/1/36/372 to JZ), the National Medical Research Council, Singapore (NMRC/CIRG/1390/2014 to JZ), and the Duke-NUS Medical School Signature Research Program funded by the Ministry of Health, Singapore.
\end{abstract}

Availability of data and materials

The datasets used and/or analyzed during the present study are available from the corresponding author on reasonable request.

\section{Authors' contributions}

AV and JZ designed the study. CLHC, SH, XX, BYT, and NV contributed to the data collection. AV, JZ, YML, SL, and HYS performed the data analysis. AV, JZ, $\mathrm{SH}, \mathrm{SL}$, and CLHC interpreted the data and wrote the manuscript. All authors read and approved the final manuscript.

\section{Ethics approval and consent to participate}

This study was conducted in accordance with the Declaration of Helsinki, and written informed consent was obtained from both the patients and the patients' caregivers. Ethics approval was granted by the National Healthcare Group Review Board of Singapore (NUHS1288/2010).

\section{Consent for publication}

Not applicable.

\section{Competing interests}

The authors declare that they have no competing interests.

\section{Publisher's Note}

Springer Nature remains neutral with regard to jurisdictional claims in published maps and institutional affiliations. 


\section{Author details}

${ }^{1}$ Centre for Cognitive Neuroscience, Neuroscience and Behavioural Disorders Program, Duke-National University of Singapore Medical School, Singapore, Singapore. ${ }^{2}$ Department of Pharmacology, Clinical Research Centre, National University Health System, National University of Singapore, Singapore, Singapore. ${ }^{3}$ Memory Aging and Cognition Centre, National University Health System, Singapore, Singapore. ${ }^{4}$ Clinical Imaging Research Centre, The Agency for Science, Technology and Research and National University of Singapore, Singapore, Singapore. ${ }^{5}$ St. Luke's Hospital, Singapore, Singapore. ${ }^{6}$ Raffles Neuroscience Centre, Raffles Hospital, Singapore, Singapore.

\section{Received: 21 March 2018 Accepted: 23 July 2018}

\section{Published online: 18 August 2018}

\section{References}

1. Chen C, Homma A, Mok VC, Krishnamoorthy E, Alladi S, Meguro K, Abe K, Dominguez J, Marasigan S, Kandiah N, et al. Alzheimer's disease with cerebrovascular disease: current status in the Asia-Pacific region. J Intern Med. 2016;280:359-74.

2. Park JH, Seo SW, Kim C, Kim SH, Kim GH, Kim ST, Jeon S, Lee JM, Oh SJ, Kim JS, et al. Effects of cerebrovascular disease and amyloid beta burden on cognition in subjects with subcortical vascular cognitive impairment. Neurobiol Aging. 2014;35:254-60.

3. Vemuri P, Lesnick TG, Przybelski SA, Knopman DS, Preboske GM, Kantarci K, Raman MR, Machulda MM, Mielke MM, Lowe VJ, et al. Vascular and amyloid pathologies are independent predictors of cognitive decline in normal elderly. Brain. 2015;138:761-71.

4. Buckner RL. Memory and executive function in aging and AD: multiple factors that cause decline and reserve factors that compensate. Neuron. 2004:44:195-208.

5. Buckner RL, Snyder AZ, Shannon BJ, LaRossa G, Sachs R, Fotenos AF, Sheline YI, Klunk WE, Mathis CA, Morris JC, Mintun MA. Molecular, structural, and functional characterization of Alzheimer's disease: evidence for a relationship between default activity, amyloid, and memory. J Neurosci. 2005;25:7709-17.

6. Greicius MD, Krasnow B, Reiss AL, Menon V. Functional connectivity in the resting brain: a network analysis of the default mode hypothesis. Proc Natl Acad Sci U S A. 2003;100:253-8.

7. Seeley WW, Crawford RK, Zhou J, Miller BL, Greicius MD. Neurodegenerative diseases target large-scale human brain networks. Neuron. 2009;62:42-52.

8. Zhou J, Greicius MD, Gennatas ED, Growdon ME, Jang JY, Rabinovici GD, Kramer JH, Weiner M, Miller BL, Seeley WW. Divergent network connectivity changes in behavioural variant frontotemporal dementia and Alzheimer's disease. Brain. 2010;133:1352-67.

9. Weiler M, de Campos BM, Nogueira MH, Pereira Damasceno B, Cendes F, Balthazar ML. Structural connectivity of the default mode network and cognition in Alzheimers disease. Psychiatry Res. 2014;223:15-22.

10. Kim HJ, Cha J, Lee JM, Shin JS, Jung NY, Kim YJ, Choe YS, Lee KH, Kim ST, Kim JS, et al. Distinctive resting state network disruptions among Alzheimer's disease, subcortical vascular dementia, and mixed dementia patients. J Alzheimers Dis. 2016;50:709-18.

11. O'Brien JT, Erkinjuntti T, Reisberg B, Roman G, Sawada T, Pantoni L, Bowler JV, Ballard C, DeCarli C, Gorelick PB, et al. Vascular cognitive impairment. Lancet Neurol. 2003;2:89-98.

12. Zhou Y, Dougherty JH Jr, Hubner KF, Bai B, Cannon RL, Hutson RK. Abnormal connectivity in the posterior cingulate and hippocampus in early Alzheimer's disease and mild cognitive impairment. Alzheimers Dement. 2008;4:265-70

13. Kim HJ, Im K, Kwon H, Lee JM, Kim C, Kim YJ, Jung NY, Cho H, Ye BS, Noh Y, et al. Clinical effect of white matter network disruption related to amyloid and small vessel disease. Neurology. 2015;85:63-70.

14. Chong JSX, Liu S, Loke YM, Hilal S, Ikram MK, Xu X, Tan BY, Venketasubramanian N, Chen CL, Zhou J. Influence of cerebrovascular disease on brain networks in prodromal and clinical Alzheimer's disease. Brain. 2017;140:3012-22.

15. Seeley WW, Menon V, Schatzberg AF, Keller J, Glover GH, Kenna H, Reiss AL, Greicius MD. Dissociable intrinsic connectivity networks for salience processing and executive control. J Neurosci. 2007;27:2349-56.

16. Uddin LQ, Kelly AM, Biswal BB, Castellanos FX, Milham MP. Functional connectivity of default mode network components: correlation, anticorrelation, and causality. Hum Brain Mapp. 2009;30:625-37.
17. Maillard P, Carmichael OT, Reed B, Mungas D, DeCarli C. Cooccurrence of vascular risk factors and late-life white-matter integrity changes. Neurobio Aging. 2015;36:1670-7.

18. Shim YS, Youn YC, Na DL, Kim SY, Cheong HK, Moon SY, Park KW, Ku BD, Lee $J Y$, Jeong $J H$, et al. Effects of medial temporal atrophy and white matter hyperintensities on the cognitive functions in patients with Alzheimer's disease. Eur Neurol. 2011;66:75-82.

19. Vemuri P, Knopman DS. The role of cerebrovascular disease when there is concomitant Alzheimer disease. Biochim Biophys Acta. 2016;1862:952-6.

20. Luchsinger JA, Brickman AM, Reitz C, Cho SJ, Schupf N, Manly JJ, Tang MX, Small SA, Mayeux R, DeCarli C, Brown TR. Subclinical cerebrovascular disease in mild cognitive impairment. Neurology. 2009;73:450-6.

21. Tosto G, Zimmerman ME, Carmichael OT, Brickman AM, Alzheimer's Disease Neuroimaging Initiative. Predicting aggressive decline in mild cognitive impairment: the importance of white matter hyperintensities. JAMA Neurol. 2014;71:872-7.

22. Tosto G, Zimmerman ME, Hamilton JL, Carmichael OT, Brickman AM, Alzheimer's Disease Neuroimaging Initiative. The effect of white matter hyperintensities on neurodegeneration in mild cognitive impairment. Alzheimers Dement. 2015;11:1510-9.

23. Greicius MD, Supekar K, Menon V, Dougherty RF. Resting-state functional connectivity reflects structural connectivity in the default mode network. Cereb Cortex. 2009;19:72-8.

24. Qiu Y, Liu S, Hilal S, Loke YM, Ikram MK, Xu X, Yeow Tan B, Venketasubramanian N, Chen CL, Zhou J. Inter-hemispheric functional dysconnectivity mediates the association of corpus callosum degeneration with memory impairment in AD and amnestic MCl. Sci Rep. 2016;6:32573.

25. Hilal S, Ikram MK, Saini M, Tan CS, Catindig JA, Dong YH, Lim LB, Ting EY, $\mathrm{Koo} \mathrm{EH}$, Cheung $\mathrm{CY}$, et al. Prevalence of cognitive impairment in Chinese: epidemiology of dementia in Singapore study. J Neurol Neurosurg Psychiatry. 2013;84:686-92.

26. Hilal S, Chai YL, Ikram MK, Elangovan S, Yeow TB, Xin X, Chong JY, Venketasubramanian N, Richards AM, Chong JP, et al. Markers of cardiac dysfunction in cognitive impairment and dementia. Medicine (Baltimore). 2015;94:e297.

27. Kapeller P, Barber R, Vermeulen RJ, Ader H, Scheltens P, Freidl W, Almkvist $O$, Moretti $M$, del Ser T, Vaghfeldt $P$, et al. Visual rating of age-related white matter changes on magnetic resonance imaging: scale comparison, interrater agreement, and correlations with quantitative measurements. Stroke. 2003;34:441-5.

28. Thong JY, Hilal S, Wang Y, Soon HW, Dong Y, Collinson SL, Anh TT, Ikram MK, Wong TY, Venketasubramanian N, et al. Association of silent lacunar infarct with brain atrophy and cognitive impairment. J Neurol Neurosurg Psychiatry. 2013;84:1219-25.

29. Wardlaw JM, Smith EE, Biessels GJ, Cordonnier C, Fazekas F, Frayne R, Lindley RI, O'Brien JT, Barkhof F, Benavente OR, et al. Neuroimaging standards for research into small vessel disease and its contribution to ageing and neurodegeneration. Lancet Neurol. 2013;12:822-38.

30. Hilal S, Saini M, Tan CS, Catindig JA, Koay WI, Niessen WJ, Vrooman HA, Wong TY, Chen C, Ikram MK, Venketasubramanian N. Cerebral microbleeds and cognition: the epidemiology of dementia in Singapore study. Alzheimer Dis Assoc Disord. 2014;28:106-12.

31. van Veluw SJ, Hilal S, Kuijf HJ, Ikram MK, Xin X, Yeow TB, Venketasubramanian $\mathrm{N}$, Biessels GJ, Chen C. Cortical microinfarcts on 3T MRI: clinical correlates in memory-clinic patients. Alzheimers Dement. 2015;11:1500-9.

32. Narasimhalu K, Ang S, De Silva DA, Wong MC, Chang HM, Chia KS, Auchus $\mathrm{AP}$, Chen C. Severity of CIND and $\mathrm{MCl}$ predict incidence of dementia in an ischemic stroke cohort. Neurology. 2009;73:1866-72.

33. Yeo D, Gabriel C, Chen C, Lee S, Loenneker T, Wong M. Pilot validation of a customized neuropsychological battery in elderly Singaporeans. Neurol J South East Asia. 1997;2:123.

34. Dubois B, Slachevsky A, Litvan I, Pillon B. The FAB: a frontal assessment battery at bedside. Neurology. 2000;55:1621-6.

35. Porteus S. The maze test and clinical psychology. Palo Alto: Pacific Books; 1959.

36. Wechsler D. Wechsler memory scale-revised. 3rd ed. San Antonio: Jovanovich; 1997.

37. Lewis R, Rennick PM. Manual for the repeatable cognitive perceptual-motor battery. Clinton Township: Axon; 1979.

38. Mack WJ, Freed DM, Williams BW, Henderson WW. Boston naming test: shortened versions for use in Alzheimer's disease. J Gerontol. 1992;47:P154-8. 
39. Isaacs B, Kennie AT. The set test as an aid to the detection of dementia in old people. Br J Psychiatry. 1973;123:467-70.

40. Smith A. Symbol digit modalities test. Los Angeles: Western Psychological Corporation; 1973.

41. Diller L, Ben-Yishay Y, Gerstman LJ. Studies in cognition and rehabilitation in hemiplegia. New York: New York University Medical Center Institute of Rehabilitation Medicine; 1974

42. Sunderland T, Hill JL, Mellow AM, Lawlor BA, Gundersheimer J, Newhouse PA, Grafman JH. Clock drawing in Alzheimer's disease. A novel measure of dementia severity. J Am Geriatr Soc. 1989;37:725-9.

43. Weschler D. Wechsler adult intelligence scale-revised. San Antonio: Harcourt Brace Jovanovich; 1981.

44. Sahadevan S, Tan NJ, Tan T, Tan S. Cognitive testing of elderly Chinese people in Singapore: influence of education and age on normative scores. Age Ageing. 1997;26:481-6

45. Ji F, Pasternak O, Liu S, Loke YM, Choo BL, Hilal S, Xu X, Ikram MK, Venketasubramanian N, Chen CL, Zhou J. Distinct white matter microstructural abnormalities and extracellular water increases relate to cognitive impairment in Alzheimer's disease with and without cerebrovascular disease. Alzheimers Res Ther. 2017;9:63.

46. Smart SD, Firbank MJ, O'Brien JT. Validation of automated white matter hyperintensity segmentation. J Aging Res. 2011;2011:391783.

47. Cox RW. AFNl: software for analysis and visualization of functional magnetic resonance neuroimages. Comput Biomed Res. 1996;29:162-73.

48. Jenkinson M, Beckmann CF, Behrens TE, Woolrich MW, Smith SM. Fsl. Neuroimage. 2012;62:782-90.

49. Ng KK, Lo JC, Lim JK, Chee MW, Zhou J. Reduced functional segregation between the default mode network and the executive control network in healthy older adults: a longitudinal study. Neuroimage. 2016;133:321-30.

50. Hong Z, Ng KK, Sim SK, Ngeow MY, Zheng H, Lo JC, Chee MW, Zhou J. Differential age-dependent associations of gray matter volume and white matter integrity with processing speed in healthy older adults. Neuroimage. 2015;123:42-50.

51. Cortese S, Imperati D, Zhou J, Proal E, Klein RG, Mannuzza S, RamosOlazagasti MA, Milham MP, Kelly C, Castellanos FX. White matter alterations at 33-year follow-up in adults with childhood attention-deficit/hyperactivity disorder. Biol Psychiatry. 2013;74:591-8.

52. Behrens TE, Berg HJ, Jbabdi S, Rushworth MF, Woolrich MW. Probabilistic diffusion tractography with multiple fibre orientations: what can we gain? Neuroimage. 2007:34:144-55.

53. Behrens TE, Woolrich MW, Jenkinson M, Johansen-Berg H, Nunes RG, Clare S, Matthews PM, Brady JM, Smith SM. Characterization and propagation of uncertainty in diffusion-weighted MR imaging. Magn Reson Med. 2003:50:1077-88.

54. Sridharan D, Levitin DJ, Menon V. A critical role for the right fronto-insular cortex in switching between central-executive and default-mode networks. Proc Natl Acad Sci U S A. 2008;105:12569-74.

55. Fishman I, Datko M, Cabrera Y, Carper RA, Muller RA. Reduced integration and differentiation of the imitation network in autism: a combined functional connectivity magnetic resonance imaging and diffusionweighted imaging study. Ann Neurol. 2015;78:958-69.

56. Hirsiger S, Koppelmans V, Merillat S, Liem F, Erdeniz B, Seidler RD, Jancke L. Structural and functional connectivity in healthy aging: associations for cognition and motor behavior. Hum Brain Mapp. 2016;37:855-67.

57. Zhou J, Gennatas ED, Kramer JH, Miller BL, Seeley WW. Predicting regional neurodegeneration from the healthy brain functional connectome. Neuron. 2012;73:1216-27.

58. Greve DN, Fischl B. Accurate and robust brain image alignment using boundary-based registration. Neuroimage. 2009;48:63-72.

59. Jbabdi S, Woolrich MW, Andersson JL, Behrens TE. A Bayesian framework for global tractography. Neuroimage. 2007;37:116-29.

60. Gong G, He Y, Concha L, Lebel C, Gross DW, Evans AC, Beaulieu C. Mapping anatomical connectivity patterns of human cerebral cortex using in vivo diffusion tensor imaging tractography. Cereb Cortex. 2009;19:524-36.

61. Bai F, Watson DR, Yu H, Shi Y, Yuan Y, Zhang Z. Abnormal resting-state functional connectivity of posterior cingulate cortex in amnestic type mild cognitive impairment. Brain Res. 2009;1302:167-74.

62. Buckner RL, Sepulcre J, Talukdar T, Krienen FM, Liu H, Hedden T, AndrewsHanna JR, Sperling RA, Johnson KA. Cortical hubs revealed by intrinsic functional connectivity: mapping, assessment of stability, and relation to Alzheimer's disease. J Neurosci. 2009;29:1860-73.
63. Qi Z, Wu X, Wang Z, Zhang N, Dong H, Yao L, Li K. Impairment and compensation coexist in amnestic $\mathrm{MCl}$ default mode network. Neuroimage. 2010;50:48-55.

64. Damoiseaux JS, Prater KE, Miller BL, Greicius MD. Functional connectivity tracks clinical deterioration in Alzheimer's disease. Neurobiol Aging. 2012:33:828.e819-30.

65. Pettersen JA, Sathiyamoorthy G, Gao FQ, Szilagyi G, Nadkarni NK, St GeorgeHyslop P, Rogaeva E, Black SE. Microbleed topography, leukoaraiosis, and cognition in probable Alzheimer disease from the Sunnybrook dementia study. Arch Neurol. 2008;65:790-5.

66. Mayda AB, Westphal A, Carter CS, DeCarli C. Late life cognitive control deficits are accentuated by white matter disease burden. Brain. 2011;134:1673-83.

67. Chan MY, Park DC, Savalia NK, Petersen SE, Wig GS. Decreased segregation of brain systems across the healthy adult lifespan. Proc Natl Acad Sci U S A. 2014;111:E4997-5006.

68. Lawrence AJ, Chung AW, Morris RG, Markus HS, Barrick TR. Structural network efficiency is associated with cognitive impairment in small-vessel disease. Neurology. 2014;83:304-11.

69. Theaud G, Dilharreguy B, Catheline G, Descotaux M. Impact of white-matter hyperintensities on tractography. In: 25th Annual Meeting of the International Society for Magnetic Resonance in Medicine (ISMRM). Honolulu: International Society for Magnetic Resonance in Medicine; 2017.

\section{Ready to submit your research? Choose BMC and benefit from:}

- fast, convenient online submission

- thorough peer review by experienced researchers in your field

- rapid publication on acceptance

- support for research data, including large and complex data types

- gold Open Access which fosters wider collaboration and increased citations

- maximum visibility for your research: over 100M website views per year

At $\mathrm{BMC}$, research is always in progress.

Learn more biomedcentral.com/submissions 